\title{
An improved method for precise genome editing in zebrafish using CRISPR-Cas9 technique
}

\author{
Eugene V. Gasanov ${ }^{1}(1) \cdot$ Justyna Jędrychowska ${ }^{1,2} \cdot$ Michal Pastor $^{1,3} \cdot$ Malgorzata Wiweger $^{1} \cdot$ Axel Methner $^{4}$. \\ Vladimir P. Korzh ${ }^{1}$
}

Received: 19 July 2020 / Accepted: 24 December 2020 / Published online: 22 January 2021

(C) The Author(s) 2021

\begin{abstract}
Current methods of CRISPR-Cas9-mediated site-specific mutagenesis create deletions and small insertions at the target site which are repaired by imprecise non-homologous end-joining. Targeting of the Cas9 nuclease relies on a short guide RNA (gRNA) corresponding to the genome sequence approximately at the intended site of intervention. We here propose an improved version of CRISPR-Cas9 genome editing that relies on two complementary guide RNAs instead of one. Two guide RNAs delimit the intervention site and allow the precise deletion of several nucleotides at the target site. As proof of concept, we generated heterozygous deletion mutants of the $k c n g 4 b$, gdapl, and ghitm genes in the zebrafish Danio rerio using this method. A further analysis by high-resolution DNA melting demonstrated a high efficiency and a low background of unpredicted mutations. The use of two complementary gRNAs improves CRISPR-Cas9 specificity and allows the creation of predictable and precise mutations in the genome of D. rerio.
\end{abstract}

Keywords CRISPR-Cas9 $\cdot$ Zebrafish $\cdot$ Precise deletion editing $\cdot$ gRNAs $\cdot$ HRM

\section{Introduction}

CRISPR-Cas9-based site-specific mutagenesis is more and more widespread in life sciences [1]. It is mainly used to inactivate (knock-out, KO) target genes by deletions and small insertions or to insert genes encoding marker proteins (knock-in, KI) at specific genomic sites of various model organisms [2]. The standard CRISPR-Cas9 KO procedure includes the design of a short guide RNA (gRNA)

Supplementary Information The online version contains supplementary material available at https://doi.org/10.1007/s1103 3-020-06125-8.

Eugene V. Gasanov

egasanov@iimcb.gov.pl; gasanovev@gmail.com

1 International Institute of Molecular and Cell Biology in Warsaw, Ks. Trojdena Str. 4, 02-109 Warsaw, Poland

2 Postgraduate School of Molecular Medicine, Medical University of Warsaw, Zwirki i Wigury Str. 61, 02-091 Warsaw, Poland

3 Institute of Biochemistry and Biophysics of Polish Academy of Sciences, Pawinskiego Str. 5a, 02-106 Warsaw, Poland

4 Institute of Molecular Medicine, University Medical Center Mainz, Langenbeckstr. 1, 55131 Mainz, Germany corresponding to the genome sequence at or close to the site of intervention and the injection of cells or developing embryos with this gRNA and Cas9 mRNA or protein. This then results in DNA double-strand breaks at the target site caused by the Cas9 nuclease and subsequent non-homologous DNA repair resulting in random mutations at the target site $[2,3]$. The creation and injection of all CRISPRCas 9 components (gRNAs and Cas9 mRNA) into zebrafish (Danio rerio) embryos is a rather simple and well-established procedure. At the same time, the screening for the desired mutants is time-consuming. Another disadvantage is the unpredictable nature of such mutagenesis. Several methods were proposed to simplify the mutagenesis and selection of mutants [3,4]. These include the usage of more than one gRNA at once to delete large segments of DNA in animal cells [5], plants [6] and invertebrates, namely Dictyostelium discoideum [7], Drosophila melanogaster and Caenorhabditis elegans [8]. CRISPR-Cas9 with two gRNAs was also used to delete from 1000 to 20,000 nucleotide base pairs (bp) in zebrafish [9].

Here we describe a significant improvement of CRISPRCas9 mutagenesis which allows the precise deletion of several nucleotides using of two gRNAs per target site. As an efficient method for mutant screening we propose the 
PCR-based high-resolution DNA melting (HRM) analysis. Using this approach, we generated two short deletion mutants of kcng $4 b$ ( $\alpha$-subunit of the voltage-gated potassium channel), a deletion mutant of gdapl (gangliosideinduced differentiation-associated protein 1), and a deletion mutant of ghitm (growth hormone-inducible transmembrane protein).

\section{Materials and methods}

\section{Animals}

Wild-type (AB line) and mutant zebrafish were maintained in the Zebrafish Facility of the International Institute of Molecular and Cell Biology in Warsaw (license no. PL14656251) according to standard procedures and ethical regulations. All experiments involving the zebrafish embryos/larvae were carried out in accordance with the Polish Laboratory Animal Science Association and Local Ethics Committee for Animal Experimentation in Warsaw (permission no. WAW2/181/2019).

To perform CRISPR-Cas9 knock-out, $2 \mathrm{nl}$ of a solution containing two gRNAs $(0.04 \mathrm{mg} / \mathrm{ml}$ of each) and Cas 9 protein $(0.2 \mathrm{mg} / \mathrm{ml}$, see below) were injected into the cytoplasm of one-cell stage wild-type AB zebrafish embryos. Fifty embryos of each, gdapl and ghitm, as well as two variants of kcng $4 b$ (variants \#1 and \#2) knock-outs were grown up after injection to 4 months to be in-crossed as the F0 founders. The phenotype of the F1 off-spring was analyzed at 1-5 dpf under a Leica M165 FC microscope. All F0 founders were fin-clipped for DNA sampling and HRM analysis. The mutation-positive ones were analyzed by DNA sequencing.

Standard single gRNA mutagenesis was performed as a control. For this, the 5'-site gRNAs of each set (all gRNAs-1 and Kcng4b-gRNA-3) were injected into 50 embryos as described above. The fish were analyzed by HRM at 5 days post fertilization (dpf) stage.

\section{gRNA synthesis}

DNA oligonucleotides encoding gRNAs with invariant adapter sequence were used, four for kcng $4 b$ and two for gdapl and ghitm: Keng4b-gRNA-1, TAATACGACTCA CTATATGAAGAGAGACTCCTTTTCTGTTTTAGAG CTAGAA; Kcng4b-gRNA-2, TAATACGACTCACTATA GTTAGCAATGGCCCAAGAAAGTTTTAGAGCTAGAA; Kcng4b-gRNA-3, TAATACGACTCACTATAAGCAGT GAGGGTTGGCTGAAGTTTTAGAGCTAGAA; Kcng4bgRNA-4, TAATACGACTCACTATACATGCAGCAGCA GTGAGGGTGTTTTAGAGCTAGAA; Gdap1-gRNA-1, TAATACGACTCACTATAGGGAGTCTACGGTGATCT
CTGTTTTAGAGCTAGAA; Gdap1-gRNA-2, TAATAC GACTCACTATAGGCTGGTATGTGGGAGTCTAGTT TTAGAGCTAGAA; Ghitm-gRNA-1, TAATACGACTCA CTATACGCGGGCAGTGTGGGCCTGAGTTTTAGAG CTAGAA; Ghitm-gRNA-2, TAATACGACTCACTATA TGTGGGCCTGACGGCGCTCTGTTTTAGAGCTAGAA (gRNA sequences are underlined, all sequences here and below have the $5^{\prime}-3^{\prime}$ direction).

For gRNA synthesis each of them was mixed with the constant oligonucleotide, containing a complementary adapter part and a $\mathrm{T} 7$ promoter sequence, Const-gRNA: AAAAGCACCGACTCGGTGCCACTTTTTCAAGTT GATAACGGACTAGCCTTATTTTAACTTGCTATTTCT AGCTCTAAAAC, both $10 \mu \mathrm{M}$, and a double-stranded product was synthesized according to the following protocol: $95^{\circ} \mathrm{C}-3$ min and 35 cycles of $95^{\circ} \mathrm{C}-15 \mathrm{~s}, 20^{\circ} \mathrm{C}-15 \mathrm{~s}$, $72{ }^{\circ} \mathrm{C}-15 \mathrm{~s}$ using PCR Mix Plus mixture (A\&A Biotechnology, Poland). The resulting DNA was purified by CleanUp Concentrator kit (A\&A Biotechnology, Poland) and verified by $2.5 \%$ agarose gel-electrophoresis. $0.2 \mu \mathrm{g}$ of DNA was used for RNA in vitro transcription by T7 RNA-Polymerase (A\&A Biotechnology, Poland) according to the manufacturer's protocol.

\section{Cas9 protein production}

DNA encoding Streptococcus pyogenes Cas9 protein with $\mathrm{N}$ - and C-terminal nuclear localization signals (NLS) was PCR amplified using PCR Mix Plus mixture (A\&A Biotechnology, Poland) and pCS2-nCas9n plasmid [11] as a template and cloned into pETM-60 vector (Novagen) using $\mathrm{NcoI}$ and $\mathrm{XhoI}$ restriction endonuclease sites. The final construct encoded an $\mathrm{N}$-terminal hexa-histidine-NusA tag followed by peptide cleavage site for tobacco etch virus (TEV) protease and C-terminal hexa-histidine (His) tag. The protein was expressed in BL21 Rosetta Escherichia coli strain (Novagen) in simplified Studier's autoinduction media [12] at $37^{\circ} \mathrm{C}$ for $4 \mathrm{~h}$ followed by $20 \mathrm{~h}$ at $20^{\circ} \mathrm{C}$. Cells were harvested by centrifugation at $4000 \times g$ for $30 \mathrm{~min}$ and stored at $-20{ }^{\circ} \mathrm{C}$.

The cell pellet was resuspended in $40 \mathrm{mM}$ Tris- $\mathrm{HCl}$ buffer (pH 7.5) containing $5 \%$ of glycerol, $0.5 \mathrm{M} \mathrm{NaCl}$, $40 \mathrm{mM}$ imidazole, $1.0 \mathrm{mM}$ phenylmethylsulfonyl fluoride (PMSF) and $2 \mathrm{mM} 2$-mercaptoethanol, then ultrasonicated and centrifugated at $10,000 \times g 4^{\circ} \mathrm{C}$ for $20 \mathrm{~min}$. The supernatant was loaded on a HisTrap FF Crude ${ }^{\circledR}$ column (GE Healthcare) equilibrated by the same buffer. After washing with $20 \mathrm{mM}$ Tris- $\mathrm{HCl}$ buffer (pH 7.5) containing 5\% of glycerol, $0.5 \mathrm{mM} \mathrm{NaCl}$ and $80 \mathrm{mM}$ imidazole, the protein was eluted by the same buffer containing $0.3 \mathrm{M}$ imidazole and the N-terminal His-NusA tag was cleaved off by TEV protease (1 $\mathrm{mg}$ per $100 \mathrm{mg}$ of Cas 9 protein) during overnight 
dialysis against $20 \mathrm{mM}$ Tris- $\mathrm{HCl}$ buffer ( $\mathrm{pH}$ 7.5) containing $5 \%$ of glycerol, $0.15 \mathrm{mM} \mathrm{NaCl}$ and $2 \mathrm{mM} 2$-mercaptoethanol. The protein solution was then clarified by centrifugation $(10,000 \times g, 10 \mathrm{~min})$ and loaded on a Q Sepharose ${ }^{\circledR}$ Fast Flow column (GE Healthcare) equilibrated by the same buffer. The flow-through was collected and dialyzed overnight against $20 \mathrm{mM}$ HEPES buffer ( $\mathrm{pH}$ 7.5) containing $0.2 \mathrm{mM} \mathrm{KCl}$ and $20 \%$ of glycerol. As a final step the protein sample was concentrated on a Vivaspin 500 centrifugal concentrator (Merk) and stored at $-80{ }^{\circ} \mathrm{C}$.

All Cas9 purification steps were controlled by spectrophotometry and by SDS-polyacrylamide gel electrophoresis.

\section{Fish mutation analysis}

The clipped fins of adult fish or 5-day-old embryos chilled on ice were placed into $30 \mu \mathrm{l}$ of $96 \%$ ethanol, heated at $80^{\circ} \mathrm{C}$ until ethanol evaporates ( 10 min) and $50 \mu \mathrm{l}$ of TE buffer (pH 8.0) were added. After $10 \mathrm{~min}$ of heating $\left(80^{\circ} \mathrm{C}\right)$ samples were chilled and incubated $3 \mathrm{~h}$ at $55^{\circ} \mathrm{C}$ with $0.5 \mathrm{mg} / \mathrm{ml}$ Proteinase $\mathrm{K}$ (Merck). After another heating $\left(10 \mathrm{~min}, 95^{\circ} \mathrm{C}\right)$ and chilling, $1 \mu \mathrm{l}$ of the solution was used as a template for PCR and HRM analysis.

The HRM analysis was performed according to the manufacturers' protocols (Roche and BioRad). PCR was performed in $10 \mu \mathrm{l}$ volume with a $0.4 \mu \mathrm{M}$ of each primer using Light Cycler 480 High-Resolution Melting Master Mix, $2 \mathrm{mM} \mathrm{MgCl}{ }_{2}$ (Roche) or using Precision Melt Supermix (BioRad). Melting curves were taken by Light Cycler 96 (Roche) or by CFX Connect Real-Time PCR System (BioRad). The following oligonucleotides were used as the primers:

For kcng4b \#1: Kg4-HRM-1f, GATGCCACTGCTAAA GAGG, and Kg4-HRM-1r, GGTTGGCTGAATGGC AGC; and for kcng $4 b \# 2$ : Kg4-HRM-2f, TGCTAACAG AAATAGCCTGC, and Kg4-HRM-2r, CTCATTAAA ACATACAGAAATCC.

For gdap 1: GDAP1-HRM-f, GGAAGCACGTATTAT CATCG; and GDAP1-HRM-r, TGCGAATGTGTGTAG TGGC.

For ghitm: GHITM-HRM-f, CGATCTGGCCGCAGT ACG; and GHITM-HRM-r, TACCAGCCAGGAGTT GCTC.

The following PCR conditions were used: pre-incubation: $95{ }^{\circ} \mathrm{C}-10 \mathrm{~min}$; 3 step amplification ( 45 cycles): $95{ }^{\circ} \mathrm{C}-10 \mathrm{~s}, 60^{\circ} \mathrm{C}-15 \mathrm{~s}, 72{ }^{\circ} \mathrm{C}-15 \mathrm{~s}$; high resolution melting: $95^{\circ} \mathrm{C}-60 \mathrm{~s}, 40^{\circ} \mathrm{C}-60 \mathrm{~s}, 65^{\circ} \mathrm{C}-1 \mathrm{~s}, 97^{\circ} \mathrm{C}-1 \mathrm{~s}$.

For the PCR and following sequencing kcng $4 b$-specific primers were used: Kcng4b-seq-fvd, CGTTCATATCAC GAACTGAAG, and Kcng4b-seq-rev, GGTAGGTCAAAT
CTTTGAAAAC; while the gdapl and ghitm fragments were amplified using the HRM primers. The following PCR conditions were used: pre-incubation: $95{ }^{\circ} \mathrm{C}-3 \mathrm{~min}$; 3 step amplification $\left(35\right.$ cycles): $95{ }^{\circ} \mathrm{C}-30 \mathrm{~s}, 58^{\circ} \mathrm{C}-30 \mathrm{~s}$, $68^{\circ} \mathrm{C}-15$ s. PCR-products were got using PCR Mix Plus mixture from A\&A Biotechnology (Poland) and sequenced from the same primers. Heterozygous sequences were analyzed using the TIDE web tool [10].

\section{Results}

We here describe an improved method of CRISPR-Cas9 genome editing to generate precise deletion mutant alleles of zebrafish kcng $4 b$, gdapl and ghitm (Fig. 1). Our method is based on the injection of two (instead of one) complementary gRNAs closely delimiting the target site along with the purified Cas9 protein into one cell $D$. rerio embryos. We found that for the 5 '-target site of kcng $4 b$ (i.e. variant \#1) 7 out of 50 (14\%) F0 founder fish carried the 5 bp deletion that matches the gap between the two gRNAs (Fig. 1). This probably represents a synergistic effect of the two gRNAs, Kcng4b-gRNA-1 and Kcng4b-gRNA-2. Two founders (4\%) had a cytosine insertion at the site targeted by Kcng $4 b$ gRNA-2. At the 3 '-targeted site of kcng $4 b$ (i.e. variant \#2), 18 of $50 \mathrm{~F} 0$ founders ( $36 \%$ ) had a deletion of $8 \mathrm{bp}$ also precisely corresponding to the gap between the two gRNAs (Fig. 1).

In the case of gdapl mutagenesis, $19 \mathrm{~F} 0$ fish (38\%) had an 11 bp deletion corresponding to the synergistic action of the Gdap1-gRNA-1 and Gdap1-gRNA-2, while 6 fish (12\%) exhibited either a 3'-shifted deletion in respect to Gdap1gRNA-1 and Gdap1-gRNA-2 delimited site or a 16-bp deletion mediated by the Gdap1-gRNA-1 alone (Fig. 1).

For the ghitm the number of mutants with the precise deletion was 22 (44\%), while 3 fish (6\%) with different deletion mutations were detected (Fig. 1). The first group of mutants corresponds to the synergistic Ghitm-gRNA-1 and Ghitm-gRNA-2 action, whereas the second, minor group represents the result of CRISPR-Cas9 mutagenesis of the Ghitm-gRNA-1 site alone.

All F0 fish carrying the CRISPR-Cas9 modification were heterozygotes. HRM detected mutations (Fig. 2) and DNA sequencing confirmed their presence.

A follow-up analysis of F1 mutant in-cross offspring of $k c n g 4 b$ variant \#1 showed the characteristic developmental defect of the kcng $4 b$ phenotype described earlier [13] for $\sim 25 \%$ of embryos (Supplementary Fig. 1). In contrast, $25 \%$ of homozygotes of the $k c n g 4 b$ variant \#2 showed a developmental phenotype different from the one described by Shen et al. [13] (Supplementary Fig. 1), which could be explained 


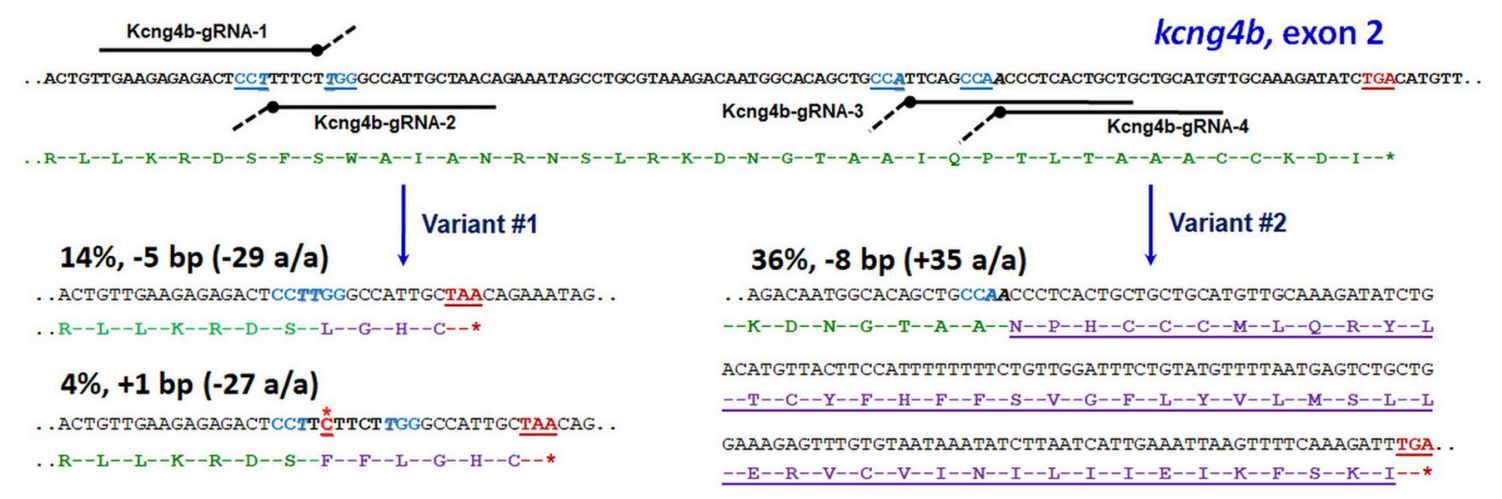

gdap1, exon 3
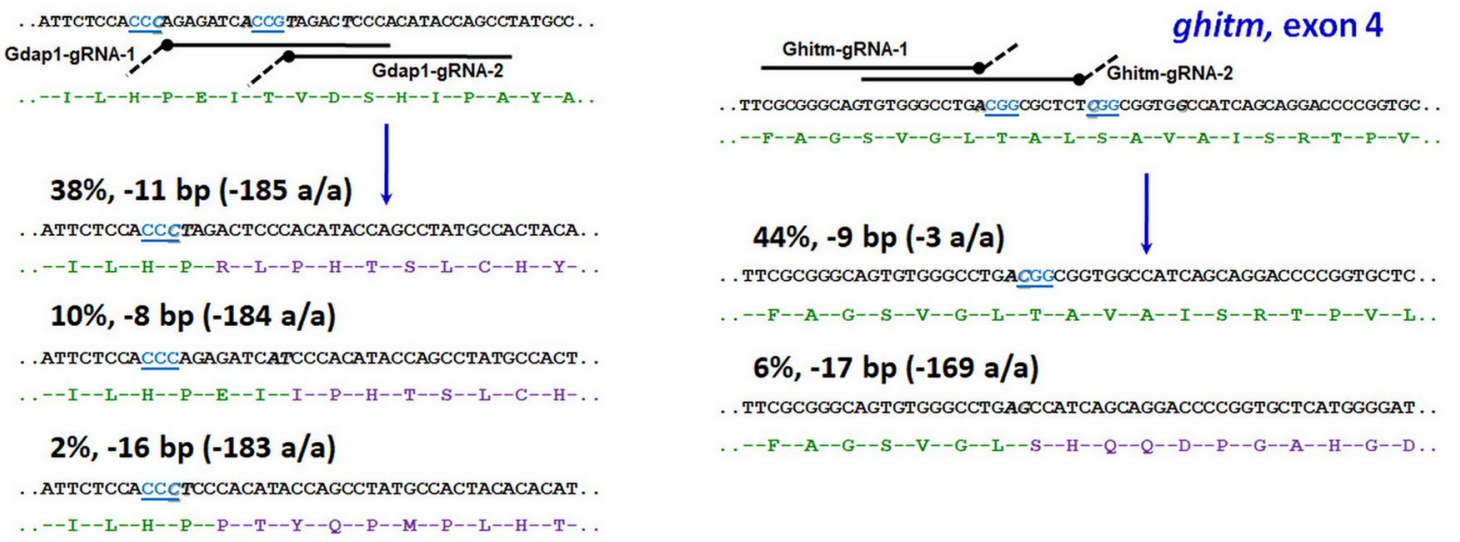

Fig. 1 The schematic view of zebrafish (D. rerio) kcng4b, gdapl and ghitm mutant generation. Stop-codons are colored red, PAMsequences are underlined and colored blue. Bold italics shows the

DNA joining nucleotides after Cas9 brakes. A wild-type translation is colored green, modified one in the mutants-purple

by the fact that the mutation does not lead to a premature truncation (Fig. 1). All kcng4b mutants exhibiting the specific phenotype described above died during the first eight days of development. This further supports the observation that all F0 founders were heterozygotes. The HRM analysis showed that surviving F1 offspring were only heterozygotes and wild type animals (Fig. 2).

Around 25\% of gdapl F0 mutant in-cross offspring exhibited a phenotype resembling the one observed after gdap 1 morpholino knock-down described previously [14] (Supplementary Fig. 1), while there was no obvious phenotype in ghitm mutant F1 offspring apparent in the first 5 days of development. An analysis of these gdapl and ghitm mutants' survival beyond this time point was precluded by the limitations imposed by our ethical regulation protocol.

In contrast to our new improved method using two gRNAs, the HRM analysis of the mutants of all three genes (kcng4b, gdapl and ghitm) generated by the traditional single gRNA CRISPR-Cas9 approach revealed a spectrum of variants. None of them represented the majority or a unified group (Fig. 3). This supports our claim that the use of two gRNAs creates more precise deletions.

\section{Discussion}

Our modification of the CRISPR-Cas9 genome editing based on two complementary gRNAs that delimit the intervention site generates a precise deletion of several nucleotides in the target gene. It makes the outcome of mutagenesis more predictable, mutant screening much easier and allows the introduction of different open reading frame (ORF) shift mutations resulting in amino acid sequence changes, mainly preliminary truncation. The precise mutagenesis was confirmed for two different sites in the kcng $4 b$ gene, and single sites in the gdapl and ghitm genes, with a different number of nucleotides deleted, in a range from 5 to 11 . This caused different ORF shifts, resulting in premature truncation in the $k c n g 4 b$ variant \#1 and gdapl, no frame shift in the case of ghitm, and prolongation of the polypeptide in the kcng $4 b$ variant \#2 (Fig. 1).

A rather negligible difference in the efficiency of generating precise deletion mutants was detected: $14 \%$ and $36 \%$ for $k c n g 4 b$ variant \#1 and \#2, 38\% for gdap1 and $44 \%$ for ghitm. These differences could be explained either by (1) specificity of gRNA action and peculiarities of 

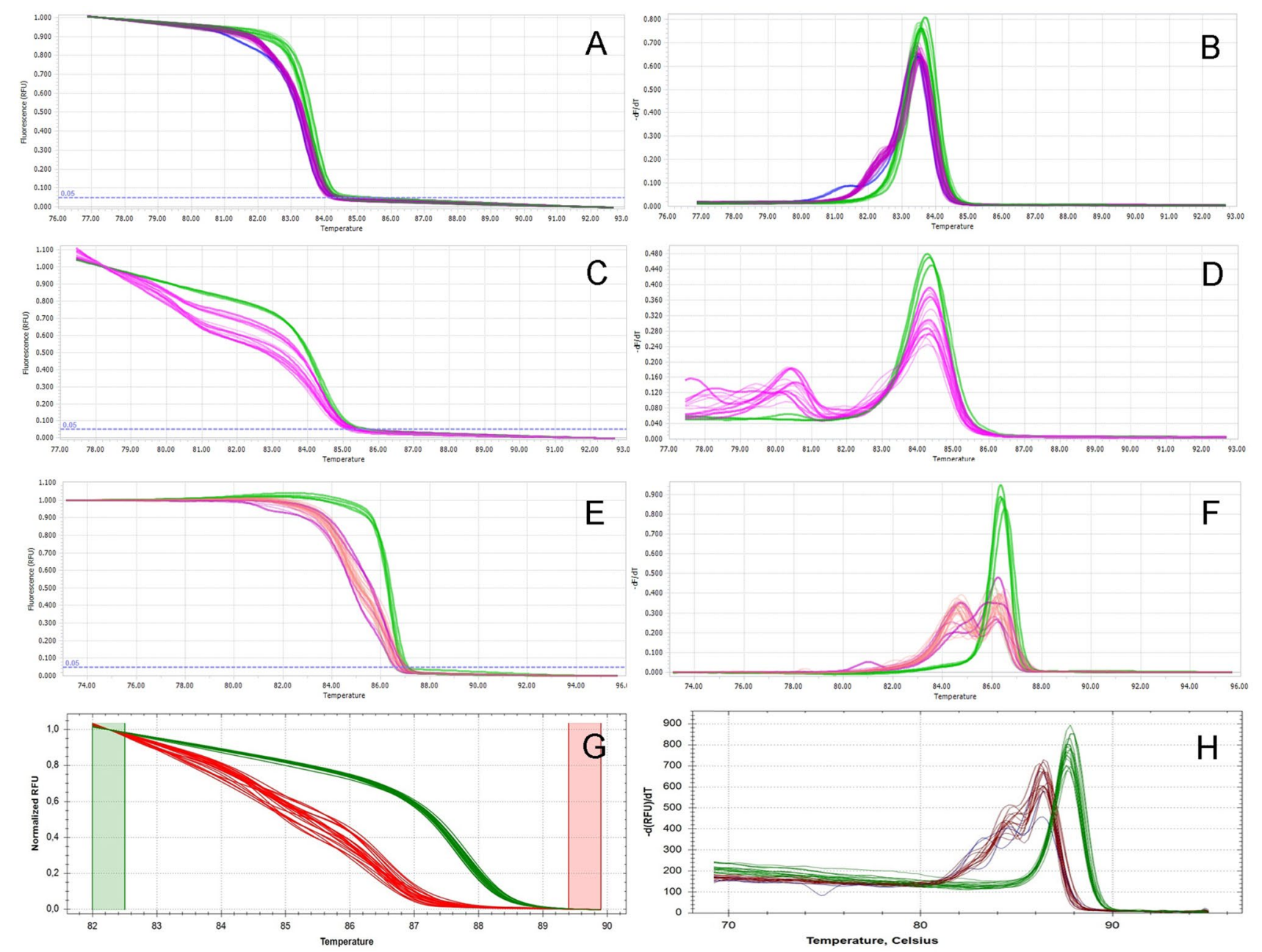

Fig. 2 The HRM analysis of the fish generated by precise two gRNAs CRISPR-Cas9 mutagenesis. (a, b) kcng $4 b$ variant \#1; (c, d) $k c n g 4 b$ variant \#2; (e, f) gdapl; (g, h) ghitm. (a, c, e, g) Normalized melting

the targeted site or (2) by mutant negative selection. The first is supported by the fact that the non-precise deletion mutants represent the minority. Moreover, the percentage of such incidental mutagenesis differs for all three genes: $4 \%$ for $k c n g 4 b$ variant \#1 with lack in variant \#2, 12\% for gdap 1, and 6\% for ghitm. The kcng $4 b$ variant \#1 incidental mutagenesis represents the insertion of a cytosine, which corresponds to the Kcng4b-gRNA-2 site-specific Cas9 action and follow-up DNA repair (Fig. 1). The ghitm incidental mutagenesis corresponds to cleavage at the GhitmgRNA-1 site. The same was detected for Gdap1-gRNA-1 (Fig. 1). All this suggests the unequal activity of the two gRNAs. Besides, the gRNA orientation may play a role. The more efficient $k c n g 4 b$ variant \#2 mutagenesis, as well as the gdapl and ghitm ones seem to rely on correspondence of both gRNAs to the same DNA strand, whereas in the less efficient $k c n g 4 b$ variant \#1 mutagenesis the gRNAs targeted opposite DNA strands (Fig. 1). Perhaps, different

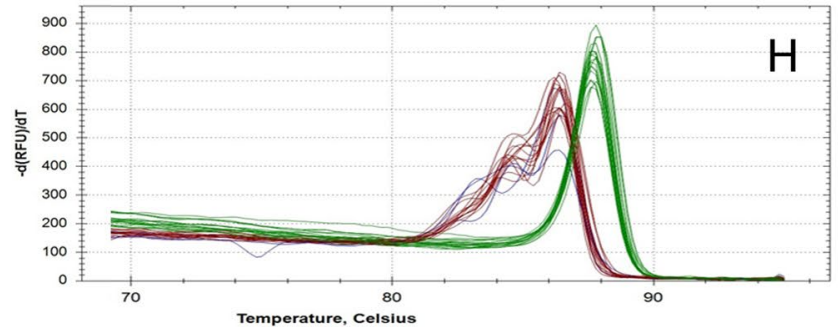

curves; (b, d, f, h) normalized melting peaks. Green-wild type; purple, red and magenta-heterozygote precise deletion mutants; blueminor mutant fractions

targeting of DNA strands may influence the effectiveness of Cas9 nuclease.

Only one insertion ( $k c n g 4 b$ variant \#1) was detected in our experiments. This supports the idea of a cooperative action of the gRNA pair even in cases of minor imprecise deletions. Presence of such incidental deletions may be caused by the DNA repair activity after a precise double gRNAs' directed cleavage.

As to the mutant negative selection and its contribution to the effectiveness of mutagenesis, it is important to note that the survival rate of the two kcng $4 b$ variants was different. This may be caused by a toxic loss-of-function of the variant \#1 mutation causing a reduction in survival even in the heterozygous state, e.g. through a deleterious effect of the mutation on spermatogenesis and/or survival of males as reported in rodents [15]. In support of a negative selection of mutants there is the highest efficiency of the ghitm mutagenesis (44\%) where deletion did not lead to ORF shift. 

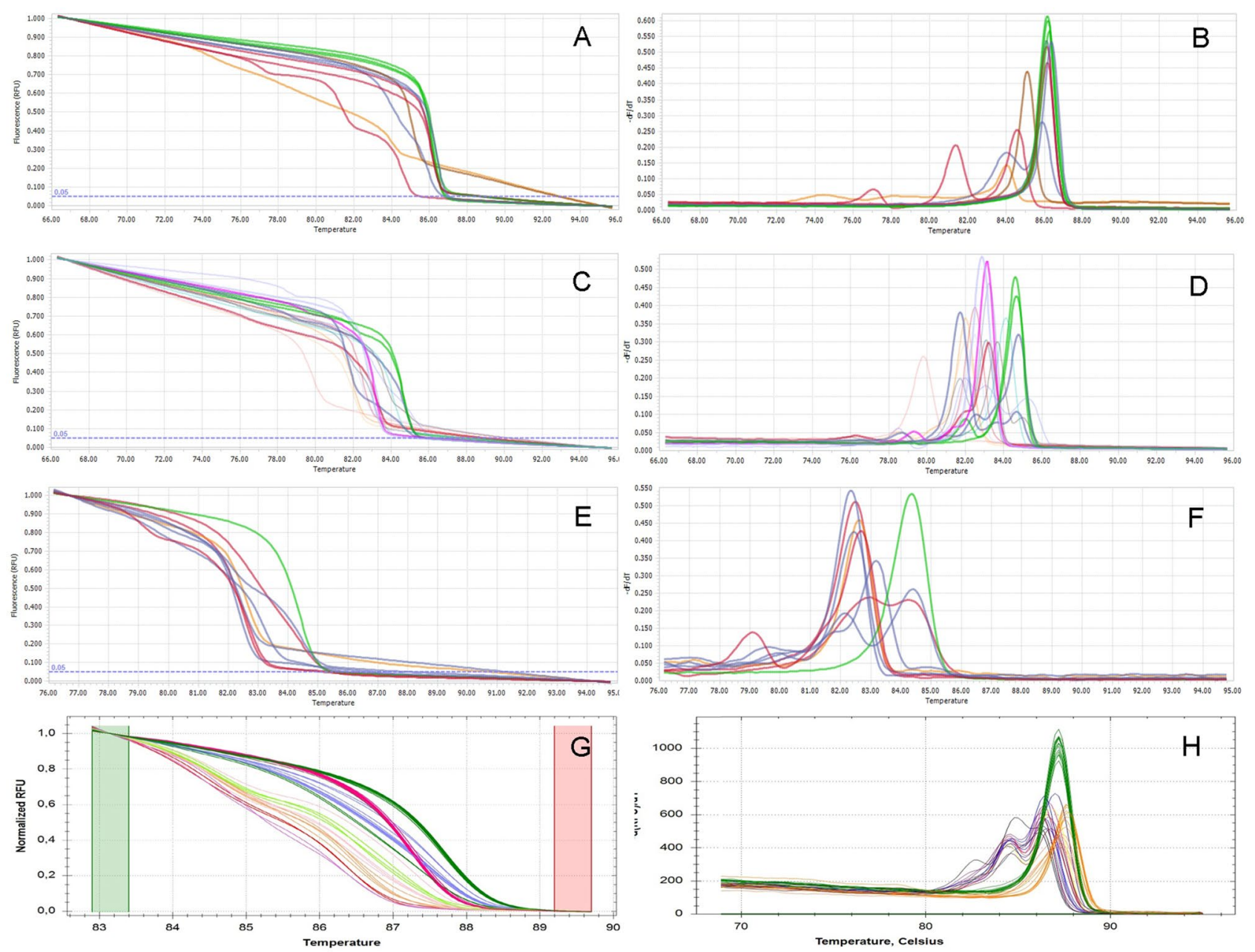

Fig. 3 The HRM analysis of the fish generated by one gRNA CRISPR-Cas9 mutagenesis. (a, b) kcng4-gRNA-1; (c, d) kcng4b-gRNA-3; (e, f) Gdap1-gRNA-1; (g, h) Ghitm-gRNA-1. (a, $\mathbf{c}, \mathbf{e}, \mathbf{g})$ Normalized melting curves; (b, d, f, h) normalized melting peaks. Green—wild type

\section{Conclusions}

Our modified version of the CRISPR-Cas9 genome editing based on the two complementary gRNAs and Cas9 protein injection allows the precise deletion of several nucleotides in the target gene. HRM-based identification of mutants is an effective, rapid, economical and reliable tool for the selection of heterozygotes.

Acknowledgements We greatly thank Prof. Jacek Kuznicki and Prof. Matthias Bochtler for their unconditional support.

Author contributions EG, AM and VK designed the project, MP cloned and purified Cas9 protein, EG and MW performed zebrafish mutagenesis, EG, JJ and MW analyzed the mutants, EG wrote the text with MP, JJ, MW, AM and VK assistance and editing.

Funding This research was supported by grants Nr 2016/21/B/ NZ3/00354 (VK) and Nr UMO-2011/02/A/NZ1/00052 (MP) from the National Science Center (Krakow, Poland) and by DFG ME1922/16-1 and ME1922/17-1 (AM). The funders had no role in study design, data collection and analysis, decision to publish, or preparation of the manuscript.

Data availability All data presented are available by request according the IIMCB Regulations.

\section{Compliance with ethical standards}

Conflict of interest The authors declare that they have no conflict of interests.

Ethical approval All experiments involving the zebrafish embryos/ larvae were carried out in accordance with the Polish Laboratory Animal Science Association rules and permission of the Local Ethics Committee for Animal Experimentation in Warsaw (permission no. WAW2/181/2019).

Consent for publication Authors agree to data publication and informed about the Publisher rules and terms.

Open Access This article is licensed under a Creative Commons Attribution 4.0 International License, which permits use, sharing, 
adaptation, distribution and reproduction in any medium or format, as long as you give appropriate credit to the original author(s) and the source, provide a link to the Creative Commons licence, and indicate if changes were made. The images or other third party material in this article are included in the article's Creative Commons licence, unless indicated otherwise in a credit line to the material. If material is not included in the article's Creative Commons licence and your intended use is not permitted by statutory regulation or exceeds the permitted use, you will need to obtain permission directly from the copyright holder. To view a copy of this licence, visit http://creativecommons .org/licenses/by/4.0/.

\section{References}

1. Lake F (2019) Putting the spotlight on: CRISPR. Biotechniques 67(2):41

2. Li M, Zhao L, Page-McCaw P, Chen W (2016) Zebrafish genome engineering using the CRISPR-Cas9 system. Trends Genet 32(12):815-827

3. Sorlien EL, Witucki MA, Ogas J (2018) Efficient production and identification of CRISPR/Cas9-generated gene knockouts in the model system Danio rerio. J Vis Exp 138:56969

4. D’Agostino Y, Locascio A, Ristoratore F, Sordino P, Spagnuolo A, Borra $\mathrm{M}$ et al (2016) A rapid and cheap methodology for CRISPR/ Cas9 zebrafish mutant screening. Mol Biotechnol 58:73-78

5. Mandal PK, Ferreira LMR, Collins R, Meissner TB, Boutwell CL, Friesen $M$ et al (2014) Efficient ablation of genes in human hematopoietic stem and effect or cells using CRISPR/Cas9. Cell Stem Cell 15:643-652

6. Do PT, Nguyen CX, Bui HT, Tran LTN, Stacey G, Gillman JD et al (2019) Demonstration of highly efficient dual gRNA CRISPR/Cas9 editing of the homologous GmFAD2-1A and GmFAD2-1B genes to yield a high oleic, low linoleic and $\alpha$-linolenic acid phenotype in soybean. BMC Plant Biol 19:311

7. Iriki H, Kawata T, Muramoto T (2019) Generation of deletions and precise point mutations in Dictyostelium discoideum using the CRISPR nickase. PLoS One 14(10):e0224128
8. Pulido-Quetglas C, Aparicio-Prat E, Arnan C, Polidori T, Hermoso T, Palumbo E et al (2017) Scalable design of paired CRISPR guide RNAs for genomic deletion. PLoS Comput Biol 13(3):e1005341

9. Hoshijima K, Jurynec MJ, Shaw DK, Jacobi AM, Behlke MA, Grunwald DJ (2019) Highly efficient CRISPR-Cas9-based methods for generating deletion mutations and F0 embryos that lack gene function in zebrafish. Dev Cell 51:1-13

10. Brinkman EK, Chen T, Amendola M, van Steensel B (2014) Easy quantitative assessment of genome editing by sequence trace decomposition. Nucleic Acids Res 42(22):e168 https://tide.deskg en.com/

11. Jao L-E, Wente SR, Chen W (2013) Efficient multiplex biallelic zebrafish genome editing using a CRISPR nuclease system. Proc Natl Acad Sci U S A 110(34):13904-13909

12. Studier FW (2005) Protein production by auto-induction in highdensity shaking cultures. Protein Expr Purif 41(1):207-234

13. Shen H, Bocksteins E, Kondrychyn I, Snyders D, Korzh V (2016) Functional antagonism of voltage-gated $\mathrm{K}^{+}$channel $\alpha$-subunits in the developing brain ventricular system. Development 143:4249-4260

14. Eijkenboom I, Vanoevelen JM, Hoeijmakers JGJ, Wijnen I, Gerards M, Faber CG, Smeets HJM (2019) A zebrafish model to study small-fiber neuropathy reveals a potential role for GDAP1. Mitochondrion 47:273-281

15. Regnier G, Bocksteins E, Marei WF, Pintelon I, Timmermans JP, Leroy JLMR et al (2017) Targeted deletion of the Kv6.4 subunit causes male sterility due to disturbed spermiogenesis. Reprod Fertil Dev 29(8):1567-1575

Publisher's Note Springer Nature remains neutral with regard to jurisdictional claims in published maps and institutional affiliations. 